\title{
Atomistic Simulations in Surface Chemistry to Interpret Scanning Probe Microscopy Images: A Short Review
}

\author{
Tiziana Musso* \\ SCS-DSM Award for best poster presentation in Computational Chemistry
}

\begin{abstract}
Atomistic simulations are a powerful tool to explain and guide experimental investigations, but there are cases where a clear correspondence is difficult to obtain. While the theoretical framework to get the static picture of the equilibrium structures in vacuum is well-established, it is challenging to correctly model them in operando conditions (at the right experimental temperature, $\mathrm{pH}$ and pressure). In this short review the main theoretical approaches are briefly presented, supported by selected case studies where the structural and dynamical properties of different systems are investigated. A successful match with the experimental data is accomplished by choosing the proper level of theory in order to describe the structure under study in the most accurate and realistic way.
\end{abstract}

Keywords: AFM · DFT · Experiments · Interface $\cdot$ Simulations $\cdot$ STM

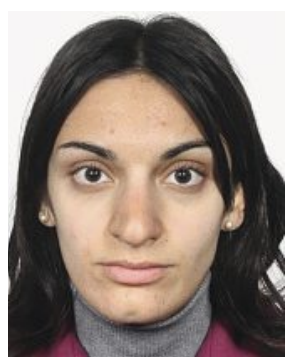

Tiziana Musso was born and raised in Italy, where she carried out her Bachelor and Master degrees studies in Physical Engineering from Politecnico di Torino. In 2016 she received her $\mathrm{PhD}$ in Engineering Physics, Theoretical and Computational Physics from Aalto University, Finland, under the guidance of Prof. Adam Foster, on Simulation of Functional Interfaces. She is currently a postdoc fellow in the group of Prof. Jürg Hutter at the University of Zürich, Switzerland. Her research focuses on ab initio simulations of heterogeneous systems for photoelectrochemical applications.

\section{Introduction}

Atomistic simulations are nowadays widely used to support experimental results, guide further investigations and even make predictions. This is particularly true in surface science, where the direct probing of phenomena at the nanoscale is not straightforward. While for an experiment it is possible to choose between different probing techniques, similarly the type and scale of the simulation can be selected. The two big general classes of atomistic simulations are quantum-mechanical and classical methods. ${ }^{[1]}$ The former class (which includes density functional theory, in short DFT) gives the most accurate results, but can be computationally expensive - it can only be applied to relatively small systems (approximately a million of atoms at best) and short time scales. Classical methods based on molecular mechanics can instead be applied to larger systems and longer time scales, but results are averaged properties and less accurate. In this case statistical mechanics is used to correlate single molecule's properties to the macroscopic thermodynamic properties of the real systems.

An approach that combines quantum-mechanical and classical methods is QM/MM (quantum mechanics/molecular mechanics),

\footnotetext{
${ }^{\star}$ Correspondence: Dr. T. Musso
}

E-mail: tiziana.musso@chem.uzh.ch

University of Zürich, Department of Chemistry, Winterthurerstrasse 190

$\mathrm{CH}-8057$ Zurich where only a part of the system, the one where more accuracy is needed, is modeled with QM; the remaining part is treated with $\mathrm{MM}$ resulting in a gain in speed.[2]

Choosing the right level of the simulations' theory method is essential to be able to compare the computed properties with the experimental ones, but still what may happen is that the simulated results do not agree with the experimental data. These 'mismatch' situations cannot be easily quantified, since usually published papers only show the 'successful matching' cases, but are indeed common. It is important to correctly interpret the experimental data and to use the right models to capture the most fundamental aspects, always being aware of the model's limitations and simplifications.

In this short review, atomistic simulations are used to match and interpret experimental scanning probe microscopy images, in terms of atomic structures and electronic properties. I will first present a few cases where atomistic simulations have successfully supported experimental data concerning the atomic structure of the investigated material and its spectroscopic properties (section 2). Methods to correctly model dynamic environments like solid/ liquid interfaces are addressed in section 3 .

\section{Structural Properties}

The system under investigation is usually a solid in contact with molecular species either in vacuum ${ }^{[3]}$ or in an aqueous media. ${ }^{[4,5]}$

It is first of all important to know the phase of the analyzed material in order to correctly model it. Characterization techniques like STM and AFM yield images with almost atomic resolution, allowing the theorists to directly compare the proposed atomistic model with the experimental images. Some codes also provide simulated STM images, that allow a direct comparison with the real STM image.

Solid/liquid interfaces are often found in experimental setups, since many processes take place in aqueous electrolytes - catalysis in UHV is not realistic. ${ }^{[6]}$ It is therefore crucial to know the properties of the involved solution, such as the $\mathrm{pH}$. Solvation models are commonly used to account for a liquid in a solid/liquid setup. They are divided in explicit, where the solvent's molecules are all included (at a great computational cost) and implicit (continuum), 
where the solvent molecules are replaced by a homogeneously polarizable medium reflecting the screening properties of the real solvent (a cheaper approach).

These types of heterostructures can be investigated with diffraction and scanning probe techniques. AFM is particularly well suited because of its atomic resolution imaging either in vacuum or in a solution, while STM cannot be easily performed in aqueous electrolytes. ${ }^{[7]}$

In the following subsections two cases are presented, where the match simulation/experiment is very good - Spijker et al. ${ }^{[5]}$ even predicted a never-observed reconstruction of the analyzed crystal.

\subsection{Phosphorus Adsorption on a Platinum (111) Surface}

Platinum is a commonly used metal in many catalytic applications, like the reduction of NOx to nitrogen and the oxidation of $\mathrm{CO}$ and hydrocarbons. Its performance is inhibited by impurities like sulfur and phosphorous that poison the catalyst. ${ }^{[8]}$ It is therefore important to study the adsorption of these impurities on the platinum surface, in order to understand how to extend the life of the catalyst. While sulfur on Pt has been extensively studied, ${ }^{[9]}$ poisoning of $\mathrm{Pt}$ by phosphorous has been only recently investigated by Heikkinen et al. ${ }^{[8]}$

In their paper, DFT calculations have been used to study the adsorption structure of molecular phosphorus on Pt(111) in UHV. In the experimental STM image it is possible to notice the particular mosaic-like arrangement that the $\mathrm{P}$ clusters adopt on the $\mathrm{Pt}$ surface (Fig. 1). From their size and shape, the authors have identified two types of $\mathrm{P}$ clusters that are plausible to be the ones on Pt. They came to this conclusion also considering the experimental $\mathrm{P}$ annealing temperature. The simulated STM image confirms this hypothesis, resulting in a perfect match with the original STM (Fig. 1). An electronic analysis highlights that some charge is being transferred from $\mathrm{P}$ clusters to the Pt surface. This interaction between the $\mathrm{P}$ clusters and the metal blocks indeed the adsorption of $\mathrm{CO}$, inhibiting the catalytic performance of Pt.

\section{2 p-Nitroaniline in Water}

Nishioka et al. ${ }^{[10]}$ performed frequency-modulation atomic force microscopy (FM-AFM) measurements in an aqueous solution of $p$-nitroaniline, an organic compound used for medical applications. Narrow-area topography images, obtained by varying the tip-surface distance to keep the frequency shift constant, highlight the presence of two configurations with non-equivalent appearances of bright spots ${ }^{[10]}$ (Fig. 2), given by the asymmetry of the AFM tip. The first topography, only rarely found, includes two spots with similar brightness, while the most commonly observed one has a combination of bright/darker spots (Fig. 2).

Large-scale MD simulations based on preliminary DFT optimizations have been used by Spijker et al..$^{[5]}$ to investigate the crystal structure of $p$-nitroaniline. Two different stable crystal configurations have been found, namely the $m$-crystal (with space group $\mathrm{P} 21 / \mathrm{m}$ ) and the $c$-crystal (space group P21/), corresponding to the rare and common AFM images. For both atomic structures, the agreement with the AFM topographic images is excellent. An analysis of water density profiles and surface hydration energies further allows to hypothesize that the actual most common $p$-nitroaniline surface is not the $c$-crystal, but a $m$-crystal with a superficial $c$-type reconstruction, even if no previous crystallographic results ever suggested this possibility, as they were focused on the crystal bulk phase. This is a perfect example of how atomistic simulations can shed light on the molecular structure of a crystal/solvent heterostructure and guide future experimental investigations.

\section{Dynamical Properties}

Once the atomic structure of the examined system is well known, dynamical processes like chemical reactions can be mod-
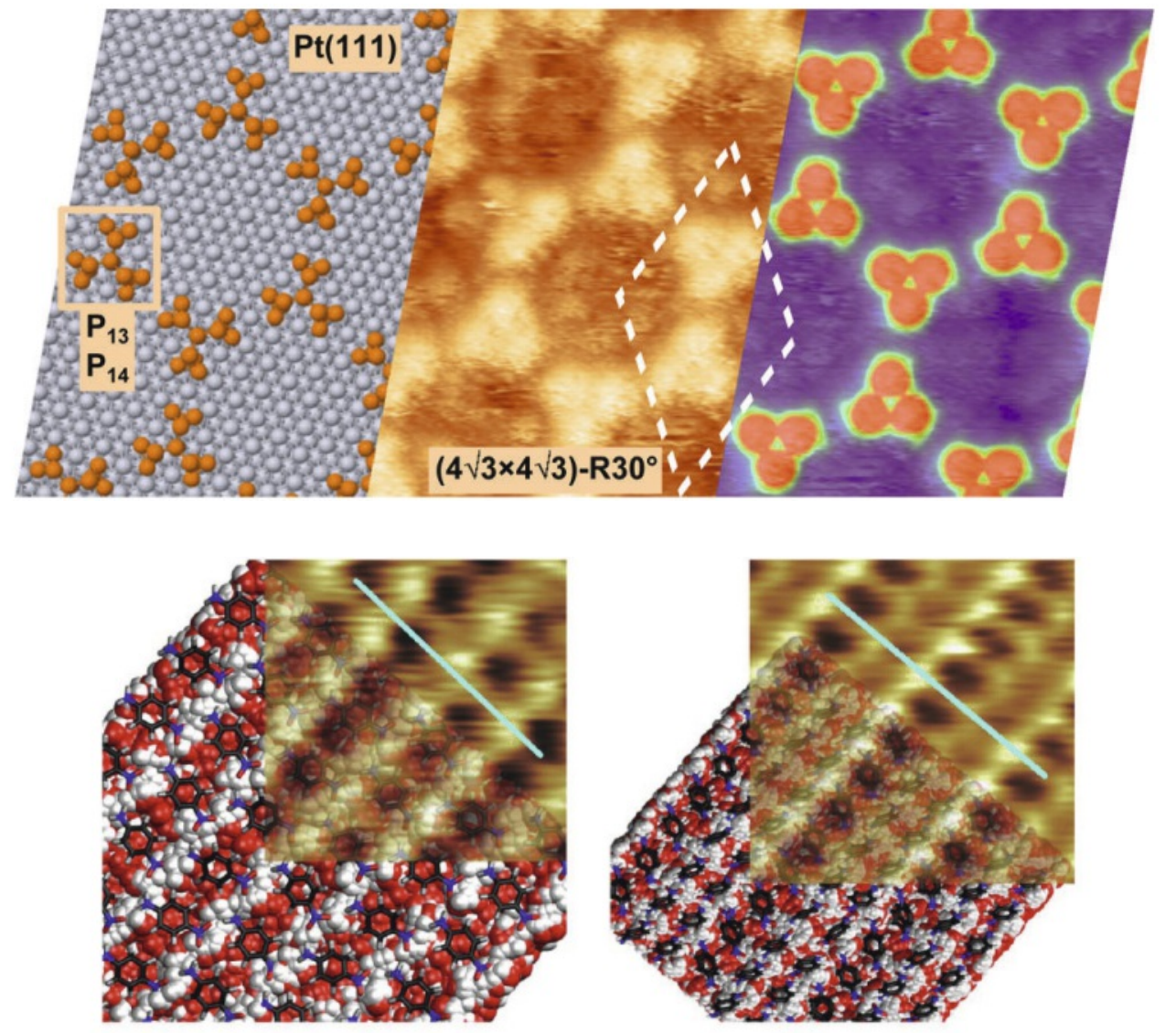

(a) AFM rare vs. MD $m$-crystal

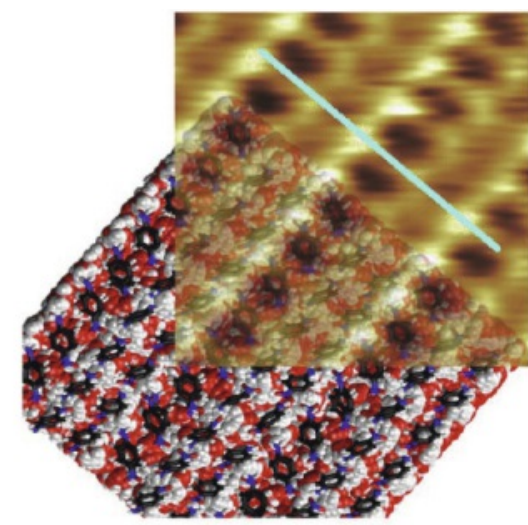

(b) AFM com. vs. MD c-crystal
Fig. 1. From left to right: top view of the unit cell of the hexagonal phosphorus pattern on top of $\mathrm{Pt}(111)$, STM image of a $\mathrm{Pt}(111)$ surface with a saturated phosphorus adlayer with sample bias and tunnelling current of $62 \mathrm{mV} / 0.42$ $\mathrm{nA}$ - phosphorus forms a hexagonal superstructure consisting of triangular clusters surrounded by circular pores (the unit cell of the superstructure is shown), simulated STM image. Reprinted with permission from ref. [8]. Copyright 2015 American Chemical Society.

Fig. 2. AFM experiments (top right) are superimposed with structures obtained from MD simulations. Left side: rare AFM is compared with the $m$-crystal of $p$-nitroaniline $\left(\mathrm{H}_{2} \mathrm{NC}_{6} \mathrm{H}_{4} \mathrm{NO}_{2}\right)$. Right side: common AFM is next to the $p$-nitroaniline $c$-crystal. Reprinted with permission from ref. [5]. Copyright 2014 American Chemical Society. 
elled through molecular dynamics (MD), where the motion of atoms is computed by applying Newton's second law to the atomic coordinates, thereby treating them classically. It is necessary to run the MD simulations for an appropriate time, in order to sample a sufficient amount of the conformational space and energy landscape. ${ }^{[11]}$ An accurate sampling of the phase space is required to get meaningful quantities that properly represent the analyzed phenomena, ${ }^{[12]}$ like the electronic structure that can be monitored along the trajectory.

In $a b$ initio MD the potential energy surface (energies, forces) is calculated at a quantum level at every optimization step, resulting in a huge computational effort feasible only for small systems and short time scales.

In section 3.1 a new approach is presented, the second-generation Car-Parrinello scheme (SGCP), that allows to obtain a substantial speed up of the $a b$ initio MD calculation. SGCP has been used to simulate complex systems like the aqueous $\mathrm{Pt}(111)$ / $\mathrm{CO}$ interface, in section 3.2. The QM/MM (quantum mechanics/ molecular mechanics) approach ${ }^{[2]}$ has instead been employed in section 3.3 to model the wetting of water on the h-BN/Rh(111) nanomesh.

In standard MD, force fields (potential functions in term of atomic coordinates) are used to get the instantaneous force on each atom. Force fields have parameters that are fitted to experimental data or high-level quantum mechanical calculations, allowing standard MD simulations to model bigger systems and longer timescales. Non-reactive empirical potentials cannot model electron-related properties like charge transfer and bond creation/breaking, while advanced atomistic potentials that include also atomic charges can. In the Tersoff potential the charges are considered fixed, while variable-charge potentials like ReaxFF and COMB allow charges to dynamically evolve, in order to model, for example, a chemical reaction. ${ }^{11]}$ The intrinsic problem of force-fields is that they are fitted to particular reference data, therefore their reliability and transferability ${ }^{[13]}$ are limited. Attempts to improve force fields are limited by their intrinsic limitations, ${ }^{[14]}$ hence novel approaches are needed.

In Section 3.4 a new type of model, FFLUX, is presented as an alternative to traditional force fields.

\section{1 h-BN/Rh(111) Nanomesh with Second Generation Car-Parrinello MD}

Hexagonal boron nitride (h-BN) has been shown ${ }^{[15]}$ to grow on top of rhodium forming the well-known nanomesh (NM), an interesting nano-structure characterized by periodic corrugation of the h-BN, given by Moiré patterns resulting from the lattice constant mismatch between the 2D material and the metal. A NM is $60 \%$ constituted by 'pore' regions, where the interaction be- tween the $\mathrm{h}-\mathrm{BN}$ and $\mathrm{Rh}$ is stronger, connected by 'wire' regions, where h-BN is more distant from the metal substrate, resulting in an overall stable periodic corrugation of the NM. Thanks to its intrinsic topology, the NM has regions with different electronic properties, giving rise to a variety of applications. ${ }^{[16]}$ Because of the complexity of NM experimental investigations, atomistic simulations are usually employed to gain valuable insights about its properties. A BN unit cell is given by $13 \times 13 h$-BN pairs on top of $12 \times 12 \mathrm{Rh}(111)$ units, hence rather large simulation cells have to be used to model the phenomena of interest. This aspect, coupled with the intrinsic complications in dealing with the metal substrate, calls for routes to overcome the current size and time-scale NM-modelling limitations of standard DFT-based MD. In ref. [17] the authors show that the second-generation Car-Parrinello scheme (SGCP), implemented in the CP2K suite of programs, ${ }^{[18]}$ results in a 17x speed up of ab initio MD simulations, compared to the standard Bohr-Oppenheimer MD scheme. SGCP constant temperature/volume (NVT) simulations of the NM shed light on the NM dynamical properties like their thermal stability.

While at $430 \mathrm{~K}$ the pores and wires are well marked, their borders become less defined at $710 \mathrm{~K}$, while at $1380 \mathrm{~K}$ it is not possible anymore to distinguish the two sites (see Fig. 3). A residence-time analysis confirms that the pores migrate over the metallic substrate with a wave-like motion that could explain the STM-resolved growth of h-BN on Rh by intact NM units. ${ }^{[19]}$ This is another example of how atomistic simulations can help understand experimental data.

\subsection{AIMD Simulations of Aqueous CO/Pt(111) Interface}

The second-generation Car-Parrinello scheme (SGCP) has also been used in the work of Lan et al., ${ }^{[20]}$ where the authors modeled the adsorption of $\mathrm{CO}$ on $\mathrm{Pt}(111)$ in the presence of liquid water at room temperature with ab initio molecular dynamics (AIMD) simulations. In electrochemical cells, platinum is commonly used as electrode material, while CO molecules are usually adopted as molecular markers, given their very specific vibrational properties. Experimental IR spectra show that $\mathrm{CO}$ bound to $\mathrm{Pt}$ in the presence of water has a characteristic red shift that is not found in dry conditions. A direct interaction of the metal with water molecules has been proposed as a possible explanation of the spectral features, but a clear explanation is lacking.

Tradition force fields are unable to capture the electronic properties of the interface, therefore AIMD simulations have been performed to get the most accurate description of the interactions between the species involved. SGCP allows to sample longer time scales, also in presence of a metal and an implicit solvent.

The authors modeled different $\mathrm{CO}$ coverages, considering the clean $\mathrm{Pt}(111)$ surface as reference. A water bilayer with an icelike
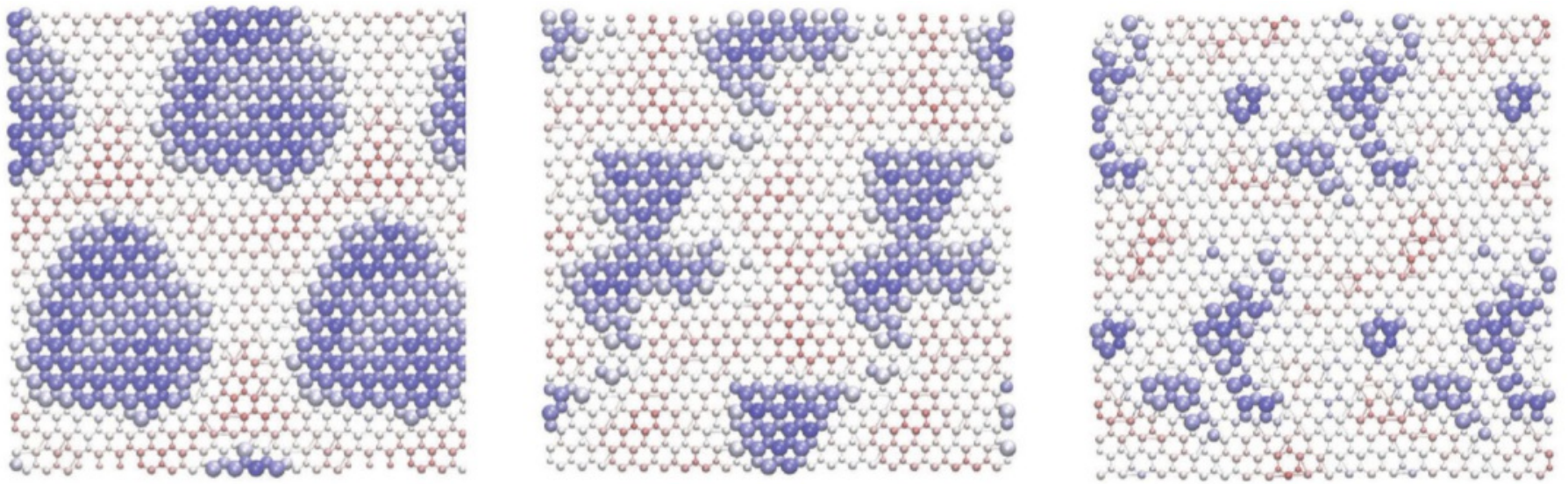

Fig. 3. Snapshots from NVT trajectories of the boron nitride nanomesh at (from left to right) $430 \mathrm{~K}, 710 \mathrm{~K}$ and $1380 \mathrm{~K}$. B and $\mathrm{N}$ atoms residing in the pore region are enlarged and blue colored, while smaller atoms in red code are part of the wire/rim regions (at least $2.7 \AA$ above the Rh surface). Reprinted with permission from ref. [17]. Copyright 2016 Springer Nature. 


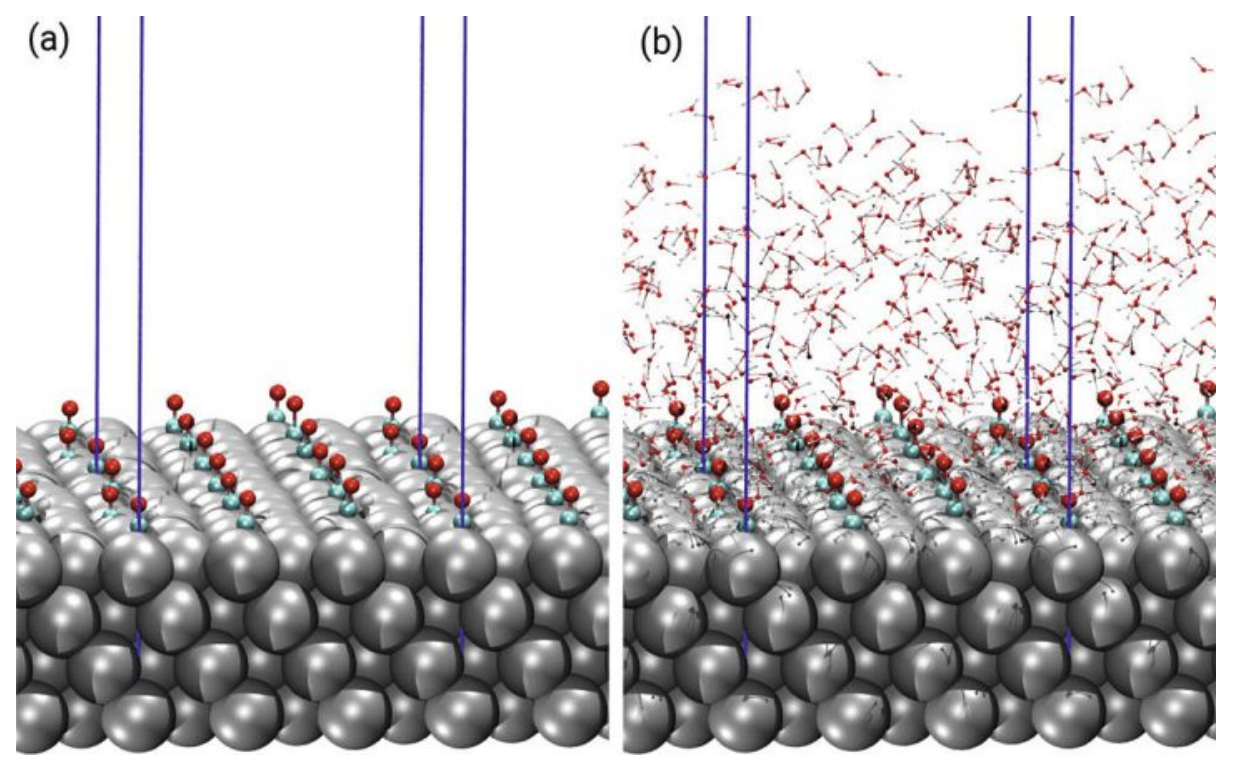

Fig. 4. Configurations of the CO monolayer adsorbed on the $\mathrm{Pt}(111)$ slab in vacuum (a) and in presence of liquid water (b). The blue lines indicate the borders of the supercell. Color code: silver represents $\mathrm{Pt}$, cyan $\mathrm{C}$, red $\mathrm{O}$, grey $\mathrm{H}$. Reprinted with permission from ref. [20]. Copyright 2018 American Chemical Society. character is formed at the surface, while liquid water is recovered after approximately $1.5 \AA$ from the surface (Fig. 4). Increasing the $\mathrm{CO}$ coverage, the structured water bilayer changes, as more $\mathrm{CO}$ molecules make it difficult for water to closely approach the $\mathrm{Pt}$ surface. A statistical analysis of long-enough trajectories allows to gather a few rare hopping events between different $\mathrm{CO}$ adsorption configurations. An accurate vibrational analysis shows that the IR red shift observed in presence of water is not caused by the interaction between water and the $\mathrm{CO}$ adsorbates through the formation of hydrogen bonds, but is an indirect effect of the water molecules of the first layer that get chemisorbed at the Pt surface, as an electronic redistribution takes place among the Pt d-band and the $\mathrm{CO}$ antibonding states. The $\mathrm{Pt}-\mathrm{C}$ bond gets looser as the $\mathrm{CO}$ or water coverage increases, allowing $\mathrm{CO}$ hopping events that result in a softer $\mathrm{CO}$ stretching mode, as experimentally observed.

The extended dynamical sampling of such a complex interface with AIMD + SGCP is therefore feasible and allows to get an accurate description of the specific thermodynamic conditions of the system under study.

\subsection{A QM/MM Model for the Wetting of Water on the h-BN/Rh(111) Nanomesh}

The wetting behavior of liquid water on functional surfaces is important for many technological applications. ${ }^{[21]}$ In their computational paper, ${ }^{[21]}$ Golze et al. modeled liquid water on top of a normal nanomesh (NM) and in a particular case where atomic $\mathrm{H}$ has been intercalated (NM-H), resulting in a flatter superficial h$\mathrm{BN}$ layer compared to the normal NM, where h-BN is corrugated. These two cases have been chosen since the wrinkling of h-BN affects the surface's functionality and hydrophilicity.

The whole system composed of water + NM/NM-H is too big to be feasibly modeled using a full quantum mechanical approach, therefore QM/MM has been used. In particular, DFT (QM) is employed to treat water, while for the substrate/molecules interactions MM is used. It is important that the modulations in the electrostatic potential (ESP) are well-reproduced by the force field used in the MM part, since the ESP is responsible to trap the water molecules in the NM pore. Usually, in normal force fields, electrostatic interactions are related to partial atomic charges. The authors came up with a fitting procedure, tailored for periodic systems, to determine the charges (called RESP charges) that will result in the correct ESP. These charges are then used in the MD simulations to correctly model the electrostatics between the NM and the water layer.

The results indeed confirm that the water molecules get trapped inside the pores in the case of the normal NM, because of the ESP effect; in the case of NM-H instead the effect is less pronounced because of the intercalated $\mathrm{H}$ atoms. This behavior has actually been confirmed by experimental STM data, ${ }^{[22]}$ where stable ice hexamers have been found only in the NM pore, not in the wire region. Concerning wettability, the NM-H surface is slightly less hydrophilic, as the contact angle of a water droplet is bigger than in the case of a regular NM.

\subsection{FFLUX}

FFLUX ${ }^{[23]}$ is a next-generation force field, where the interactions between the atoms are described quantum mechanically, overcoming the shortcomings of traditional force fields since no fitted potentials are required. Even if to date it has only been tested on small organic molecules, ${ }^{[23]}$ it shows promising performances, as it can correctly reproduce their ground state to a $<0.01 \mathrm{eV}$ accuracy with respect to reference DFT values using the B3LYP functional.

In FFLUX the atoms have a quantum description since the interacting quantum atoms (IQA) scheme is used to topologically partition the atoms (Fig. 5) in a parameters and orbitals-free way. IQA is as a matter of fact a quantum chemical topology (QCT) scheme that partitions the wave function only using the gradient of the electron density. The kriging (or Gaussian process regression) machine learning method is then used to obtain potentials able to reproduce the potential energy surface (PES) of the target molecules, giving information on barrier heights and stationary points.

The energy and structures of the molecules' most stable configurations are well reproduced by FFLUX. An increase in the number of the training set conformations employed to train the FLLUX model will result in further accuracy improvements, but it will lead to a higher computational cost. Since the FFLUX perfor-

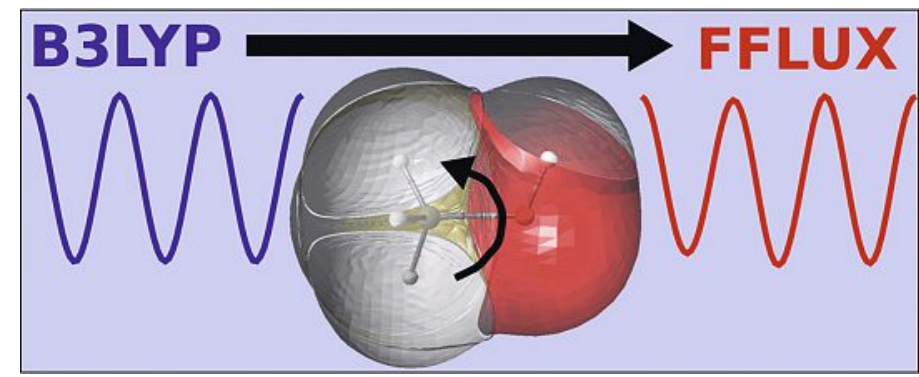

Fig. 5. Schematic showing the topological partition of a molecule based on the interacting quantum atoms (IQA) scheme. Reprinted with permission from ref. [23]. Copyright 2018 American Chemical Society. 
mance is affected by how the ensemble of conformations used to construct the model is generated and by its size, future efforts will focus on finding ways to reduce the dimension of the conformation ensemble needed to correctly model the system. ${ }^{[23]}$

\section{Conclusions}

Thanks to supercomputers, nowadays computational chemists can model the atomic structures of the systems under study and their dynamical behavior, even predicting features that might be experimentally confirmed. MD simulations with the SGCP allow to sample the dynamics of molecules adsorbed on a surface under specific thermodynamic conditions, while with the QM/MM approach, a part of the system can be treated classically (MD) and a part at the quantum (DFT) level. Solid/liquid interfaces remain challenging to simulate in operando conditions, but new approaches to get rid of the limitations of classical force-fields are currently investigated.

In the end, to get good agreement between experimental scanning probe microscopy and simulated data, it is important to use the most appropriate theoretical framework that best describes the experimental setup and that is most convenient also in terms of computational cost.

\section{Acknowledgements}

The author is grateful to DSM and the Swiss Chemical Society for being awarded the best poster presentation award. Thanks also to MARVEL and the University of Zürich through the LightChEC program for their generous support, and to PD Dr. Marcella Iannuzzi for useful discussions.
[1] T. Musso, PhD Thesis, Aalto University, 1799-4934, 2016.

[2] H. Lin, D. G. Truhlar, Theor. Chem. Acc. 2006, 117, 185.

[3] Y. Gurdal, S. Luber, J. Hutter, M. Iannuzzi, Phys. Chem. Chem. Phys. 2015, $17,22846$.

[4] E. Holmström, P. Spijker, A. S. Foster, Proc. R. Soc. A 2016, 472, 20160293.

[5] P. Spijker, T. Hiasa, T. Musso, R. Nishioka, H. Onishi, A. S. Foster, J. Phys. Chem. C 2014, 118, 2058.

[6] M. Müllner, J. Balajka, M. Schmid, U. Diebold, S. F. L. Mertens, J. Phys. Chem. C 2017, 121, 19743.

[7] M. Nielsen, M. E. Björketun, M. H. Hansen, J. Rossmeisl, Surf. Sci. 2015, $631,2$.

[8] O. Heikkinen, H. Pinto, G. Sinha, S. K. Hämäläinen, J. Sainio, S. Öberg, P. R. Briddon, A. S. Foster, J. Lahtinen, J. Phys. Chem. C 2015, 119, 12291.

[9] A. K. Neyestanaki, F. Klingstedt, T. Salmi, D. Y. Murzin, Fuel 2004, 83, 395.

[10] R. Nishioka, T. Hiasa, K. Kimura, H. Onishi, J. Phys. Chem. C 2013, 117, 2939.

[11] M. Nemec, D. Hoffmann, J. Chem. Theory Comput. 2017, 13, 400.

[12] K. Gordiz, D. J. Singh, A. Henry, J. Appl. Phys. 2015, 117, 045104.

[13] F. Sato, S. Hojo, H. Sun, J. Phys. Chem. A 2003, 107, 248.

[14] T. Hynninen, T. Musso, A. S. Foster, Modelling Simul. Mater. Sci. Eng. 2016, 1.

[15] M. Corso, W. Auwärter, M. Muntwiler, A. Tamai, T. Greber, J. Osterwalder, Science 2004, 303, 217.

[16] M. Iannuzzi, F. Tran, R. Widmer, T. Dienel, K. Radican, Y. Ding, J. Hutter, O. Gröning, Phys. Chem. Chem. Phys. 2014, 16, 12374.

[17] T. Musso, S. Caravati, J. Hutter, M. Iannuzzi, Eur. Phys. J. B 2018, 91, 1824

[18] J. Hutter, M. Iannuzzi, F. Schiffmann, J. VandeVondele, WIREs Comput. Mol. Sci. 2013, 4, 15.

[19] G. Dong, E. B. Fourré, F. C. Tabak, J. W. M. Frenken, Phys. Rev. Lett. 2010, 104, 096102.

[20] J. Lan, J. Hutter, M. Iannuzzi, J. Phys. Chem. C 2018, 122, 24068.

[21] D. Golze, J. Hutter, M. Iannuzzi, Phys. Chem. Chem. Phys. 2015, 17, 14307.

[22] H. Ma, T. Brugger, S. Berner, Y. Ding, M. Iannuzzi, J. Hutter, J. Osterwalder, T. Greber, ChemPhysChem 2010, 11, 399.

[23] Z. E. Hughes, J. C. R. Thacker, A. L. Wilson, P. L. A. Popelier, J. Chem. Theory Comput. 2018, 15, 116. 